after 28 days in pots in the greenhouse. Stock boosted with fertilizer in the nursery prior to lifting "regenerated roots more strongly" than did the normal stock. The less carefully handled seedlings "could not respond to fertilizer in the pots. Gently handled high nutrition seedlings were already regenerating roots prolifically without pot fertilizer. Roughly handled seedlings from the low nutrition nursery beds regenerated almost no new roots and in the first two months in the forest approximately $10 \%$ of such seedlings died." FRI concludes that nutritional boosting in the nursery is more economic than field fertilization after outplanting and "will help to get seedlings rooting quickly". But "the benefit will be cancelled if the seedllings are roughly handled at lifting."

Many other items of interest are reported by the Production Forestry Division as well as the Protection Forestry and Wood Technology Divisions.

This continues the excellent series of FRI annual reports. The illustrations are attractive and well chosen, the layout is good, and the text informative. The only improvement that I would suggest is that it would be useful to identify the researcher(s) along with the work described in the text, even though the staff listing does indicate fields of work.

\section{R.F. Sutton}

Forestry. Report of the Nova Scotia Royal Commission on Forestry. Halifax, Nova Scotia, 113 p., 1984. Enquiries to Royal Commission on Forestry, Dr. John Connor, Chairman, 1740 Granville Street, Box 698, Halifax, Nova Scotia, B3J 2 T9.

The 50 or so recommendations include the following: Silviculture - "The Commission recommends that a silviculture programme, within the context of comprehensive management plans, and based upon scientific knowledge and professional experience, be established in Nova Scotia"; and Herbicides - "The Commis-
Écologie et Dendrométrie dans le sudouest du Québec : Étude de douze secteurs forestiers. Z. Majcen, Y. Richard, et M. Ménard. Ministère de l'Énergie et des ressources, Service de la recherche, (Terre de Forêts), Québec, 333 p., 1984, Mémoire No. 85, ISBN 2-550-11041-2. In French with English summary. Original abstracts, French and English.

A partir de 452 relevés, on a reconnu 34 associations, 36 sous-associations, 7 groupements, 9 variantes et 7 faciès. Cette étude phytosociologique est liée à celle des sols et d'autres caractères d'habitat: pierrosité, épaisseur et nature du dépôt meuble, drainage, pente et exposition. Différentes variables dendrométriques servent à exprimer le rendement pour comparer les groupements forestiers et les secteurs entre eux et les classer selon ces différences. Ressortent les avantages et la nécessité d'une classification écologique en aménagement des forêts: plus cet aménagement est intensif, plus la connaissance du milieu doit être poussée.

From 452 samples, 34 associations, 36 sub-associations, 7 groups, 9 variants and 7 facies were recognized. This phytosociological study is related to that of soils and other environmental factors: stoniness, depth and nature of surface deposit, drainage, slope and exposition. Different mensuration data are used to determine yield in order to compare forest groups and sectors with one another and to classify them along their differences. Results bring out the advantages and the necessity of ecological classification in forest management: as the latter becomes more intensive, knowledge of the environment become more important.

sion is convinced that registered herbicides, used according to label instructions do not constitute a health hazard either to those directly involved, or to those who might inadvertently come into contact with them, and recommends that the use of federally registered herbicides be recognized as a necessary and safe tool in forestry."
A Compendium of Danish Forest Machinery, 1984 Edition. Pieter D. Kofman (Ed.), Danish Forest Machine Manufacturers, Copenhagen, 72 p., 1984, ISBN 87-87798-35-2.

Products from 27 firms are described and illustrated in this edition of the compendium. Additional information may be obtained from: Mr. Pieter D. Kofman, Amalievej 20, DK 1875 Copenhagen V, Denmark.

Résultats et Impacts des Projets de Recherche en Économie Forestière, 1970-1982. Bilan sommaire par thème de recherche. Québec Ministère de l'Énergie et des Ressources, Service de la recherche (Terres et Forêts), Division de l'Économie Forestière, Québec, $67 \mathrm{p}$. 1983, ISBN 2-550-10612-1.

Ce document présente sous forme conçise un bilan des principaux résultats et impacts des projets de recherche entrepris ou réalisés par la Division d'Économie forestière du Service de la recherche du M.E.R. depuis sa création.

\title{
Institut Forestier du Canada
}

\section{National Officers 1985-86 Bureau de direction national}

$\begin{array}{ll}\text { Toovey, J.W. } & \text { President } \\ \text { Carrow, J.R. } & \text { 1st Vice-President } \\ \text { Kayll, A.J. } & \text { 2nd Vice-President } \\ \text { Clark, J.D. } & \text { Past President } \\ \text { Place, I.C.M. } & \text { Editor } \\ \text { Redmond. D.R. } & \text { Production Manager } \\ \text { Bourchier, R.J. } & \text { Executive Director }\end{array}$

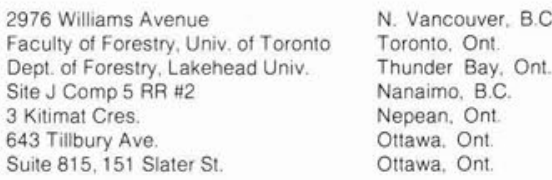

Ottawa Ont

Ottawa, Ont.

V7K 1 Z6

M5S 1 A 1

P7B 5E1

V9R $5 \mathrm{~K} 2$

K1P $5 \mathrm{H} 3$
Home Business

(807) $345-2121$

(613) $829-2105$

(613) $728-8721$

(613) $234-2242$

\section{Section Officers 1985-86 / Responsables régionaux}

SECTION ALGONQUIN SECTION

Haddon, B.D. Chairperson. CIF

Algonquin Section

Algonquin Section

Brand, D.G. Secretary. CIF Algonquin Section

SECTION CARIBOO CHILCOTIN SECTION

Routledge, Doug Chairperson, CIF Cariboo Chilcotin Section

Dufresne Keith Director, CIF Cariboo Chilcotin Section

Penny, Barry S. Secretary CIF Cariboo Chilcotin Section
Petawawa Nt1. For. Inst

Box 335

Petawawa Nti. For Inst.

$\%$ W Fraser TBR, Box 4360

Esler Rd. N. Box 11, R.R. \#1 Box 4360
Chalk River, Ont

Renfrew. Ont.

Chalk River. Ont

KOJ IJO

K7V 4A4

KOJ 1 JO

Williams Lake, BC

Williams Lake, B.C.

Williams Lake, B.C
V2G 2V4

V2G $2 P 1$

V2G 2 V 4 


\section{SECTION CARIBOO SECTION}

\begin{tabular}{|c|c|c|}
\hline $\begin{array}{l}\text { Trenaman. R.G. } \\
\text { Logan. Brian } \\
\text { Donnelly. Francis }\end{array}$ & $\begin{array}{l}\text { Secretary, CIF } \\
\text { Director, CIF } \\
\text { Secretary, CIF }\end{array}$ & $\begin{array}{l}\text { Cariboo Section } \\
\text { Cariboo Section } \\
\text { Cariboo Section }\end{array}$ \\
\hline $\begin{array}{l}\text { SECTION CENTRAI } \\
\text { Graham, H.D. } \\
\text { Smyth. J.H. } \\
\text { MacNaughton, R.L. }\end{array}$ & $\begin{array}{l}\text { L ONTARIO SECT } \\
\text { Chairperson, CIF } \\
\text { Director, CIF } \\
\text { Secretary, CIF }\end{array}$ & $\begin{array}{l}\text { ON } \\
\text { Central Ontario Section } \\
\text { Central Ontario Section } \\
\text { Central Ontario Section }\end{array}$ \\
\hline $\begin{array}{l}\text { SECTION CHAMPL } \\
\text { Pouliot, Luc } \\
\text { Bergevin, Réjean } \\
\text { Lefebvre, Marjolaine }\end{array}$ & $\begin{array}{l}\text { AIN SECTION } \\
\text { Chairperson, CIF } \\
\text { Director, CIF } \\
\text { Secretary, CIF }\end{array}$ & $\begin{array}{l}\text { Champlain Section } \\
\text { Champlain Section } \\
\text { Champlain Section }\end{array}$ \\
\hline $\begin{array}{l}\text { SECTION KOOTEN } \\
\text { Bailey. Jack } \\
\text { Bailey, Jack } \\
\text { Rounsville. Dennis }\end{array}$ & $\begin{array}{l}\text { AY SECTION } \\
\text { Chairperson, CIF } \\
\text { Director, CIF } \\
\text { Secretary, CIF }\end{array}$ & $\begin{array}{l}\text { Kootenay Section } \\
\text { Kootenay Section } \\
\text { Kootenay Section }\end{array}$ \\
\hline $\begin{array}{l}\text { SECTION LAKE OF } \\
\text { Ferguson, Bruce } \\
\text { Suomu, Len } \\
\text { Laurie McCulligh }\end{array}$ & $\begin{array}{l}\text { THE WOODS SE } \\
\text { Chairperson, CIF } \\
\text { Director, CIF } \\
\text { Secretary, CIF }\end{array}$ & $\begin{array}{l}\text { TION } \\
\text { Lake of the Woods Section } \\
\text { Lake of the Woods Section } \\
\text { Lake of the Woods Section }\end{array}$ \\
\hline $\begin{array}{l}\text { SECTION MANITOE } \\
\text { Jonas, W.E. } \\
\text { Wotton, D.L. } \\
\text { Keenan, Vince }\end{array}$ & $\begin{array}{l}\text { BA SECTION } \\
\text { Chairperson, CIF } \\
\text { Director, CIF } \\
\text { Secretary. CIF }\end{array}$ & $\begin{array}{l}\text { Manitoba Section } \\
\text { Manitoba Section } \\
\text { Manitoba Section }\end{array}$ \\
\hline $\begin{array}{l}\text { SECTION MARITIM } \\
\text { Stewart. J.V. } \\
\text { Hoyt. J. Stephen } \\
\text { Mann, A.G. }\end{array}$ & $\begin{array}{l}\text { E SECTION } \\
\text { Chairperson CIF } \\
\text { Director, CIF } \\
\text { Secretary, CIF }\end{array}$ & $\begin{array}{l}\text { Maritime Section } \\
\text { Maritime Section } \\
\text { Maritime Section }\end{array}$ \\
\hline $\begin{array}{l}\text { SECTION NEWFOU } \\
\text { Case, Boyd } \\
\text { Munro. John }\end{array}$ & $\begin{array}{l}\text { NDLAND SECTIC } \\
\text { Chairperson. CIF } \\
\text { Director, CIF }\end{array}$ & $\begin{array}{l}\text { Newfoundland Section } \\
\text { Newfoundland Section }\end{array}$ \\
\hline
\end{tabular}

Alexander, William Secretary CIF Newfoundland Section

\section{SECTION NORTHERN ONTARIO SECTION}

$\begin{array}{lll}\text { Galloway. Rob } & \text { Chairperson, ClF } & \text { Northern Ontario Section } \\ \text { Galloway. Rob } & \text { Director, CIF } & \text { Northern Ontario Section }\end{array}$

Cameron, Richard Secretary, CIF Northern Ontario Section

\section{SECTION NORTHWESTERN ONT. SECTION}

Swant, GIn Chairperson, CIF Northwestern Ont. Section

Benson. C Director, ClF Northwestern Ont Section

Zago, Teresa Secretary. CIF Northwestern Ont. Section

\section{SECTION NOVA SCOTIA SECTION}

$\begin{array}{lll}\text { Jackson, Peter } & \text { Chairperson, CIF } & \text { Nova Scotia Section } \\ \text { Bowers, Charles } & \text { Director, CIF } & \text { Nova Scotia Section }\end{array}$

Bowers, Charles Secretary, CIF Nova Scotia Section

SECTION OKANAGAN SECTION

$\begin{array}{lll}\text { Don Couch } & \text { Chairperson, CIF } & \text { Okanagan Section } \\ \text { Birzins, Paul } & \text { Director, CIF } & \text { Okanagan Section } \\ \text { Annand, Robert } & \text { Secretary, CIF } & \text { Okanagan Section }\end{array}$

Annand, Robert Secretary, CIF Okanagan Section

\section{SECTION ORLEANS SECTION}

$\begin{array}{lll}\text { Gauvin, Jacques } & \text { Chairperson, CIF } & \text { Orleans Section } \\ \text { Gauvin, Jacques } & \text { Director, CIF } & \text { Orleans Section }\end{array}$

Boivin, Florentn Secretary, CIF Orleans Section

\section{SECTION OTTAWA VALLEY SECTION}

$\begin{array}{lll}\text { Carlson, Les } & \text { Chairperson, CIF } & \text { Ottawa Valley Section } \\ \text { Clarke, Mike } & \text { Director, CIF } & \text { Ottawa Valley Section }\end{array}$

Clarke, Mike Oitawa Valley Section

\section{SECTION PACIFIC SECTION}

$\begin{array}{llll}\text { Willcocks, A.J. } & \text { Chairperson, CIF } & \text { Pacific Section } & \text { Box } 5066 \\ \text { Willcocks, A.J. } & \text { Director, CIF } & \text { Pacific Section } & \text { Box } 5066 \\ \text { Symon, Margaret } & \text { Secretary CIF } & \text { Pacific Section } & \text { Box } 5000\end{array}$

Symon, Margaret Secretary CIF Pacific Section

\section{SECTION ROCKY MOUNTAIN SECTION}

Robertson, Sharon Chairperson, CIF Rocky Mountain Section

Pratley, Howard Director, CIF Rocky Mountain Section

Archibald, H. Secretary, CIF Rocky Mountain Section

$\begin{array}{lll}\text { Thompson, J. } & \text { Chairperson, CIF } & \text { Saskatchewan Section } \\ \text { Stoddart, R.J. } & \text { Director, CIF } & \text { Saskatchewan Section }\end{array}$

Frey, G.E. Secretary, CIF Saskatchewan Section

\section{SECTION SKEENA SECTION}

$\begin{array}{llll}\text { Raven, D.J. } & \text { Chairperson, CIF } & \text { Skeena Section } & \text { Box } 3602 \\ \text { Raven, D.J. } & \text { Director, CIF } & \text { Skeena Section } & \text { Box } 3602\end{array}$

Doubt, J. Secretary, CIF Skeena Section $\quad$ Box 2574

\section{SECTION SOUTHERN ONTARIO SECTION}

Martin, J.A.S. Chairperson, CIF Southern Ontario Section

Martin, J.A.S. Director, CIF Southern Ontario Section

Pickering, Susan Secretary, CIF Southern Ontario Section

\section{SECTION VANCOUVER ISLAND SECTION}

Walker, J. Chairperson, CIF Vancouver Island Section

Muller, R. Secretary, CIF Vancouver Island Section

\section{SECTION VANCOUVER SECTION}

$\begin{array}{lll}\text { Strang, R.M. } & \text { Chairperson, CIF } & \text { Vancouver Section } \\ \text { Strang. R.M. } & \text { Director, CIF } & \text { Vancouver Section }\end{array}$

$\begin{array}{lll}\text { Strang, R.M. } & \text { Director, CIF } & \text { Vancouver Section } \\ \text { Wooding, P. } & \text { Secretary, CIF } & \text { Vancouver Section }\end{array}$
4399 Eaglenest Cres

Laurentian Dr.

101-1595 Fifth Ave.

1184 Queen St. E.

CFS Box 490

P.O. Box 490

100 Alexis-Nihon Blvd Ste. 300

2212 Rosemon

246 rue St. Charles

Box 501

Box 501

Crestbrook For. Ind. Box

Min. Nat: Resources, Box 309 Box 623

Min. Nat. Resources, Box 309

Box 2175

70 'Brien Cres

Box 457

Cdn. For Serv. P.O. Box 4000

Comp. 17. Site 11

Marit. Forest. Ranger Schl., R.R.\#10

CFS BIdg. 304, Box 6028

52 Highland Drive

CFS BIdg. 304, Box 6028

22 Hillcrest Dr.

22 Hillcrest Dr.

133 Main St.

168 Ibbetson Str. 12

624 Rosewood $\mathrm{Cr}$.

Lakehead Univ. Sch. of Forestry

Nova Scotia Forest Industries

R.R. \#3, St. Andrews

R.R. $\# 3$, St Andrews

Site 18A. Comp. 8. R.R \#6

Kalamalka Research Stn.

C.30, McLeod Rd. N.

4541 Des Bosquets

4541 Des Bosquets

142 Bon Air

1676 Ortona Ave.

619 Eddy St.

R.R. \#5, Box 75

Res. Eval. Br. DENR. 9945108 St.

Univ of Alta. Dept. Forest Sci.

Res. Eval, Br. DENR. 9945108 St.

1378 Lacroix Cr.

660 Buchanan Dr.

Dept. Pks. ' Ren. Res., Box 3003 Sanford Flem. College. Box 8000

MNR 3626 Whitney Blk. Queen's Pk.

B.C. For. Prds. Lid

1746 Westlock R.R. \#5

R.R. \#1,7390 Walton Mtn. Rd

For. Res. 7671 Alderbridge Way 3 For. Res. 7671 Alderbridge Way 3 Cdn. For. Prds., Box 49420 Bentall
Sanford Flem. College, Box 8000
Prince George, B.C.

Prince George, B.C.

$\mathrm{V} 2 \mathrm{~N} 1 \mathrm{Z3}$

V2L 3 LN

Sault Ste Marie, Ont. P6A 2E6

Sault Ste Marie, Ont P6A 5M7

Sault Ste. Marie. Ont. P6A 5M7

St. Laurent, Qué $\quad H 4 M$ 2N8

Montreal, Qué. H2G 1 T5

Dorion, Qué, J7V 2L7

Creston, B.C. VOB $1 \mathrm{GO}$

Creston, B.C. VOB $1 \mathrm{GO}$

Cranbrook. B.C. V1C $4 \mathrm{~T} 7$

(604) 428-2729

Sioux Lookout, Ont. POV 2TO

Ear Falls, Ont. POV 1TO

Sioux Lookout

POV 2TO

The Pas, Man, R9A 1 Z8

Winnipeg. Man. R3R 1 M5

Pine Falls. Man ROE $1 \mathrm{MO}$

Fredericton, NB E3B 5P7

R.R. $\# 8$ Fredericton, N.B. E3B 5W5

Fredericton, N.B. E3B $6 \mathrm{H} 6$

St. John's. Nfld. A1C $5 \times 8$

Wedgewood Park, Nild. A1E 3 Y 2

St. John's, Nild. A1C $5 \times 8$

Kirkland Lake, Ont. P2N 3M4

Kirkland Lake, Ont. P2N 3M4

Kirkland Lake. Ont. P2N 3G2

Thunder Bay, Ont. P7A 7J9

Thunder Bay, Ont. P7E 2R7

Thunder Bay, Ont. P7B 5B1

Port Hawkesbury, N.S. BOE 2VO

Antigonish. NS. $\mathrm{ROH} 1 \mathrm{XO}$

$\begin{array}{ll}\text { Antigonish, N.S. } & R O H 1 \times O \\ \text { Antigonish. N.S. } & \mathrm{ROH} 1 \times 0\end{array}$

Vernon, B.C $\quad$ V1T 6 Y5

$\begin{array}{ll}\text { Vernon, B.C. } & \text { V1T } 6 Y 5 \\ \text { Vernon, B.C. } & \text { V1B 2C7 }\end{array}$

R.R. \#2, Armstrong, B.C. VOE $1 B 0$

St. Augustin, P.Q GOA $3 E O$

St. Augustin, P.Q. GOA $3 E O$

St. Rédempteur, P. Q. GOS $3 B 0$

Ottawa. Ont. K2C 1W7

Buckingham. Qué. J8L 127

Otlawa. ont. K1G 3 N3

Woss, B.C

VON $3 P O$

Woss, B.C.

VON 2 RO

Edmonton. Alta.

T5K 2 G9

Edmonton, Alta.

T6G $2 \mathrm{H} 1$

Edmonton, Alta.

T5K 2 G6

Prince Albert Sask

Prince Albert, Sask.

S6V $6 R 8$
S6V $5 Z 6$

Prince Albert, Sask. S6V 6G1

Smithers. B.C

Smithers, B.C.

Smithers, B.C

VOJ 2 NO

VOJ 2 NO

VOJ 2 NO

Lindsay, Ont

Lindsay. Ont.

K9V $5 E 6$

K9V $5 E 6$

M7A 1 W3

(807) 737-2734 (807) 737-1140 (807) $222-2452$

(807) 737-3770 (807) 737-1140

(204) $945-7081$

506) $454-3893$ (506) 452-3638 (506) 472-1378 (506) 453-2165 (506) 366-3108 (506) 454-4363

(709) 772.4682

(709) $772-2318$

(705) $642-3222$

(705) $567-3372$

(705) 642-3222

(807) $344-8464$

(807) $622-5690(807) 345-2121$

(807) $345-8329(807) 345-2121$

(902) 625-0455 (902) 625-2460

(604) 542-4311

(604) 545-3598 (604) 545-2285 (604) 546-8711

(819) $997-3350$
(819) $986-2487$

(613) $997-3350$

(604) $974-5551$ (604) $974-5551$

(604) $956-4062$ (604) $956-4411$

(403) 427-7222

(403) 432-2820

(403) 427-7222

(306) $922-2344$

(604) 847-4611 (604) 847-7505 (604) $847-4611(604) 847-7505$ (604) 846-9281 (604) 847-7504

(705) $324-9144$ (705) 361.0810 (705) $324-9144$ (705) $361-0810$ (416) $445-0653$ (416) 965-2785

Honey Moon Bay, B.C. VOR 1 Yo Duncan, B.C. V9L 4T6

(604) 748-5266 (604) 745-3324

Duncan, $\mathrm{B}$.

V9L $1 \mathrm{M} 3$

(604) $748-5123$ (604) 746-5123

Vancouver, B.C.

v $6 \times 129$

Vancouver, B.C.

v $6 \times 129$

V7K $1 B 5$
(604) 273-6548

(604) $273-6548$ 